\title{
Quantum information transfer with superconducting flux qubits coupled to a resonator
}

\author{
Chui-Ping Yang \\ Department of Physics, Hangzhou Normal University, Hangzhou, Zhejiang 310036, China
}

(Dated: March 22, 2022)

\begin{abstract}
We propose a way for implementing quantum information transfer with two superconducting flux qubits, by coupling them to a resonator. This proposal does not require adjustment of the level spacings or uniformity in the device parameters. Moreover, neither adiabatic passage nor a secondorder detuning is needed by this proposal, thus the operation can be performed much faster when compared with the previous proposals.
\end{abstract}

PACS numbers: 03.67.Lx, 42.50.Dv, 85.25.Cp

Introduction.-Cavity QED with superconducting qubits including superconducting charge qubits, phase qubits and flux qubits have been considered as one of the most promising candidates for quantum information processing. Superconducting qubits have the features such as design flexibility, large scale integration, and compatibility to conventional electronics [1-3]. A cavity or resonator acts as a "quantum bus" which can mediate long-distance, fast interaction between distant superconducting qubits [4].

In recent years, there is much interest in quantum information transfer (QIT). One of its applications is as follows. When performing quantum information processing in a practical system, after a step of processing is completed, we need to transfer the state of the operation qubit to the memory qubit for storage. Then we need to transfer the state from the memory qubit to the operation qubit when a further step of processing is needed. Thus, it is an interesting topic to realize quantum state transfer between qubits. Experimentally, QIT has been demonstrated with superconducting phase qubits and transmon qubits in cavity QED $[5,6]$. However, to the best of our knowledge, no experimental demonstration of QIT with superconducting flux qubits in cavity QED has been reported.

Several theoretical methods have been proposed for implementing QIT with flux qubits (e.g., SQUID qubits) or charge-flux qubits based on cavity QED technique [7-12]. These methods are useful for the physical realization of QIT with flux qubits in cavity QED. However, these methods have some disadvantages. For instances: (i) the method presented in [8] requires adjustment of the level spacings of the devices during the operation; (ii) the methods proposed in [7,9-11] require slowly changing the Rabi frequencies to satisfy the adiabatic passage; and (iii) the approach introduced in [12] requires a second-order detuning to achieve an off-resonant Raman coupling between two relevant levels. Note that the adjustment of the level spacings during the operation is undesirable and also may cause extra decoherence. In addition, when the adiabatic passage or a second-order detuning is applied, the operation becomes slow (the operation time required for the information transfer is on the order of one microsecond to a few microseconds $[7,12])$.

In this paper, we present a way for implementing QIT with two flux qubits coupled to a superconducting resonator. As shown below, this proposal has the following advantages: (a) the qubits are not required to have identical level spacings, therefore superconducting devices, which often have considerable parameter nonuniformity, can be used; (b) the method does not require adjustment of the level spacings of each qubit during the operation, thus decoherence caused by tuning the level spacings is avoided; (c) neither adiabatic passage nor a second-order detuning is needed, thus the operation is speeded up (as shown below, the operation time for the information transfer is on the order of ten nanoseconds).

Basic theory.-The flux qubits throughout this paper have three levels $|0\rangle,|1\rangle$, and $|2\rangle$, which form a $\Lambda$-type configuration depicted in Fig. 1. The transition between the two lowest levels is forbidden due to the optical selection rules [13] or weak via increasing the potential barrier between the two levels $|0\rangle$ and $|1\rangle$ [14-16]. The qubits with this three-level structure could be a radio-frequency superconducting quantum interference device (rf SQUID) consisting of one Josephson junction enclosed by a superconducting loop [see Fig. 2(a)], or a superconducting device with three Josephson junctions enclosed by a superconducting loop [Fig. 2(b)]. When the loop is flux-biased properly and/or the device parameters are chosen appropriately, the desired level structure shown in Fig. 1 is available. For flux qubits, the two logic states of a qubit are represented by the two lowest levels $|0\rangle$ and $|1\rangle$.

A). Qubit-resonator-pulse resonant Raman coupling. Consider a flux qubit coupled to a single-mode resonator and driven by a classical microwave pulse (Fig. 1). Suppose that the resonator mode is coupled to the $|0\rangle \leftrightarrow|2\rangle$ transition but decoupled (highly detuned) from the transition between any other two levels. In addition, assume that the classical microwave pulse is coupled to the $|1\rangle \leftrightarrow|2\rangle$ transition but decoupled from the transition between any 


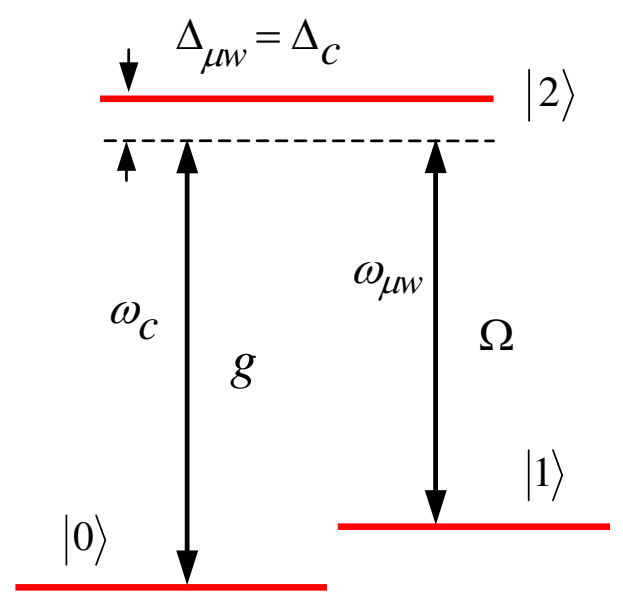

FIG. 1: (Color online) Qubit-resonator-pulse resonant Raman coupling. The tunneling between the two lowest levels is forbidden or weak, such that quantum information for each qubit, encoded in the two lowest levels, can be stored for a long time.

other two levels. The Hamiltonian of the system can thus be written as

$$
H=\sum_{l} E_{l} \sigma_{l l}+\omega_{c} a^{+} a+\hbar g\left(a^{+} \sigma_{02}^{-}+\text {H.c. }\right)+\hbar \Omega\left(e^{i \omega_{\mu w} t} \sigma_{12}^{-}+\text {H.c. }\right)
$$

where $a^{+}$and $a$ are the photon creation and annihilation operators of the resonator mode with frequency $\omega_{c} ; g$ is the coupling constant between the resonator mode and the $|0\rangle \leftrightarrow|2\rangle$ transition of the qubit; $\Omega$ is the Rabi frequency of the pulse and $\omega_{\mu w}$ is the frequency of the pulse; $\sigma_{02}^{-}=|0\rangle\left\langle 2\left|, \sigma_{12}^{-}=\right| 1\right\rangle\langle 2|$, and $\sigma_{l l}=|l\rangle\langle l|(l=0,1,2)$.

Suppose that the resonator mode is off-resonant with the $|0\rangle \leftrightarrow|2\rangle$ transition, i.e., $\Delta_{c}=\omega_{02}-\omega_{c} \gg g$, and the pulse is off-resonant with the $|1\rangle \leftrightarrow|2\rangle$ transition, i.e., $\Delta_{\mu w}=\omega_{12}-\omega_{\mu w} \gg \Omega$ (Fig. 1), where $\omega_{02}$ is the $|0\rangle \leftrightarrow|2\rangle$ transition frequency and $\omega_{12}$ is the $|1\rangle \leftrightarrow|2\rangle$ transition frequency. Under this condition, the level $|2\rangle$ can be adiabatically eliminated [17]. Thus, for $\Delta_{c}=\Delta_{\mu w}$, the effective Hamiltonian in the interaction picture is $[12,18]$

$$
H_{\mathrm{eff}}=-\hbar\left[\frac{\Omega^{2}}{\Delta_{\mu w}} \sigma_{11}+\frac{g^{2}}{\Delta_{c}} a^{+} a \sigma_{00}+\frac{\Omega g}{\Delta_{c}}\left(a^{+} \sigma_{01}^{-}+\text {H.c. }\right)\right],
$$

where $\sigma_{01}^{-}=|0\rangle\langle 1|$. The first two terms in Eq. (2) are ac-Stark shifts of the levels $|1\rangle$ and $|0\rangle$ induced by the pulse and the resonator mode, respectively; while the last two terms in Eq. (2) are the familiar Jaynes-Cummings interaction, describing the resonant Raman coupling between the two lowest levels $|0\rangle$ and $|1\rangle$, which results from the cooperation of the resonator mode and the pulse.

For the case of $\Omega=g$, the initial states $|0\rangle|1\rangle_{c}$ and $|1\rangle|0\rangle_{c}$ of the system, under the Hamiltonian (2), evolve as follows

$$
\begin{aligned}
|0\rangle|1\rangle_{c} & \rightarrow e^{i g^{2} t / \Delta_{c}}\left[\cos \left(g^{2} t / \Delta_{c}\right)|0\rangle|1\rangle_{c}-i \sin \left(g^{2} t / \Delta_{c}\right)|1\rangle|0\rangle_{c}\right], \\
|1\rangle|0\rangle_{c} & \rightarrow e^{i g^{2} t / \Delta_{c}}\left[-i \sin \left(g^{2} t / \Delta_{c}\right)|0\rangle|1\rangle_{c}+\cos \left(g^{2} t / \Delta_{c}\right)|1\rangle|0\rangle_{c}\right],
\end{aligned}
$$

where $|0\rangle_{c}$ and $|1\rangle_{c}$ are the vacuum state and the single-photon state of the resonator mode, respectively. The state $|0\rangle|0\rangle_{c}$ remains unchanged under the Hamiltonian (2).

The coupling strength $g$ may vary with different qubits due to non-uniform device parameters and/or non-exact placement of qubits in the resonator. Therefore, in the information transfer operation below, we will replace $g$ by $g_{a}$ and $g_{b}$ for qubits $a$ and $b$, respectively. Accordingly, we will replace $\Delta_{c}$ by $\Delta_{c}^{a}$ and $\Delta_{c}^{b}, \Delta_{\mu w}$ by $\Delta_{\mu w}^{a}$ and $\Delta_{\mu w}^{b}$, and $\Omega$ by $\Omega_{a}$ and $\Omega_{b}$ for qubits $a$ and $b$, respectively.

B). Qubit-pulse resonant interaction. Consider a three-level flux qubit driven by a classical microwave pulse. Suppose that the pulse is resonant with the transition $|i\rangle \leftrightarrow|j\rangle$ of the qubit but decoupled from the transition between any two other levels. Here, $|i\rangle$ is the lower energy level. The interaction Hamiltonian in the interaction picture is given by

$$
H_{I}=\hbar\left(\widetilde{\Omega} e^{i \phi}|i\rangle\langle j|+\text { H.c. }\right) \text {, }
$$




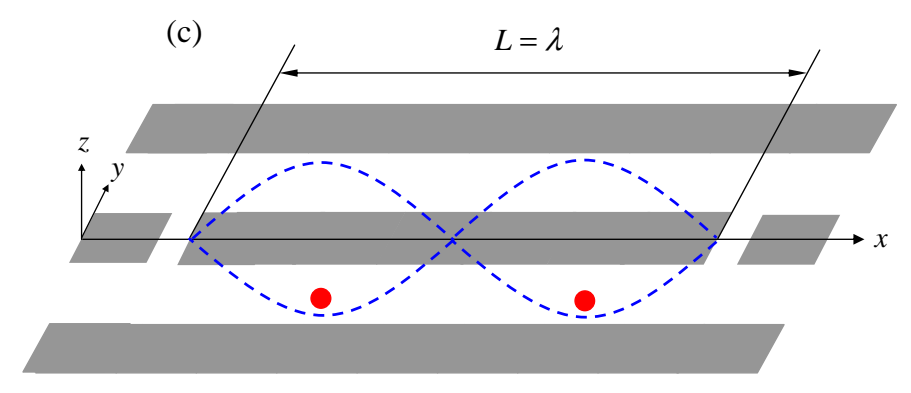

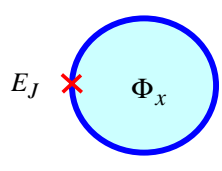

(a)

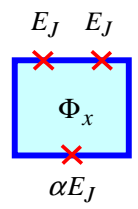

(b)

FIG. 2: (Color online) (a) An rf SQUID consisting of one Josephson junction enclosed by a superconducting loop. (b) A superconducting device with three Josephson junctions enclosed by a loop. The level spacings of a flux qubit shown in Fig. 1 can be adjusted by changing external magnetic flux $\Phi_{x}$ applied to the loop. Here, $E_{J}$ is the Josephson junction energy, and $0<\alpha<1$. (c) Sketch of the setup for two superconducting flux qubits (red circles) and a (grey) standing-wave quasi-onedimensional coplanar waveguide resonator. The two blue curved lines represent the standing wave magnetic field, which is in the $z$ direction. Each qubit could be an rf SQUID shown in (a) or a superconducting device with three Josephson junctions shown in (b). The qubits are placed at antinodes of the resonator mode to achieve maximal qubit-resonator coupling constants. The superconducting loop of each qubit is located in the plane of the resonator between the two lateral ground planes (i.e., the $x-y$ plane). $\lambda$ is the wavelength of the resonator mode and $L$ is the length of the resonator.

where $\widetilde{\Omega}$ and $\phi$ are the Rabi frequency and the initial phase of the pulse, respectively. Based on the Hamiltonian (4), it is straightforward to show that a pulse of duration $t$ results in the following rotation

$$
\begin{aligned}
& |i\rangle \rightarrow \cos \widetilde{\Omega} t|i\rangle-i e^{-i \phi} \sin \widetilde{\Omega} t|j\rangle, \\
& |j\rangle \rightarrow \cos \widetilde{\Omega} t|j\rangle-i e^{i \phi} \sin \widetilde{\Omega} t|i\rangle .
\end{aligned}
$$

The transition frquency $\omega_{i j}$ between the two levels $|i\rangle$ and $|j\rangle$ may be different for qubits $a$ and $b$ due to their nonidentical level spacings. Thus, in the following, we will replace $\omega_{i j}$ by $\omega_{i j}^{a}$ and $\omega_{i j}^{b}$ for qubits $a$ and $b$, respectively.

Quantum information transfer.- Let us now consider two superconducting flux qubits $a$ and $b$ coupled to a resonator [Fig. 2(c)]. Each qubit has a $\Lambda$-type three-level configuration as depicted in Fig. 1. The quantum information of a qubit is encoded by the two lowest levels $|0\rangle$ and $|1\rangle$. Suppose that qubit $a$ is the original carrier of quantum information, which is in an arbitrary state $\alpha|0\rangle+\beta|1\rangle$. The QIT from qubit $a$ to qubit $b$ initially in the state $|1\rangle$ is described by

$$
\left(\alpha|0\rangle_{a}+\beta|1\rangle_{a}\right)|1\rangle_{b} \rightarrow|1\rangle_{a}\left(\alpha|0\rangle_{b}+\beta|1\rangle_{b}\right) .
$$

From Eq. (6), one can see that this process can be done via a transformation that satisfies the following truth table:

$$
\begin{aligned}
|0\rangle_{a}|1\rangle_{b} & \rightarrow|1\rangle_{a}|0\rangle_{b}, \\
|1\rangle_{a}|1\rangle_{b} & \rightarrow|1\rangle_{a}|1\rangle_{b} .
\end{aligned}
$$

To realize the transformation (7), suppose that the resonator mode is off-resonant with the $|0\rangle \leftrightarrow|2\rangle$ transition of each qubit (with a detuning $\Delta_{c}^{a}=\omega_{02}^{a}-\omega_{c}$ for qubit $a$ while $\Delta_{c}^{b}=\omega_{02}^{b}-\omega_{c}$ for qubit $b$ ) but highly detuned (decoupled) from the transition between any other two levels of each qubit. Note that this condition can be readily achieved by prior adjustment of the level spacings of the qubits before the operation (e.g., this is doable for superconducting qubits by varying the external flux applied to the superconducting loop $[14-16,19,20])$. In addition, we assume that the resonator mode is initially in the vacuum state $|0\rangle_{c}$.

We find that the transformation (7) can be implemented through the following operations: 
Qubit a

(a)

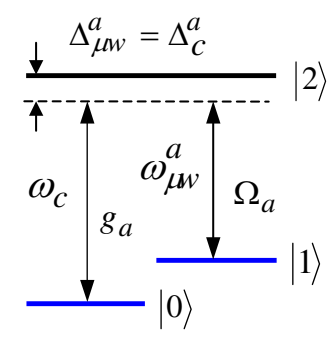

(b)

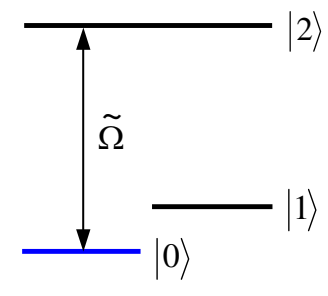

(c)

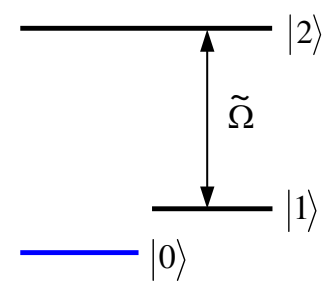

(d)

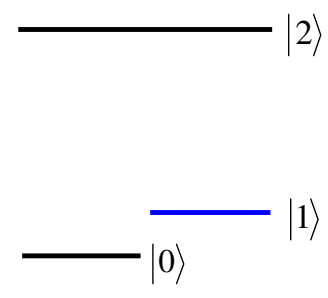

(a')

Qubit b

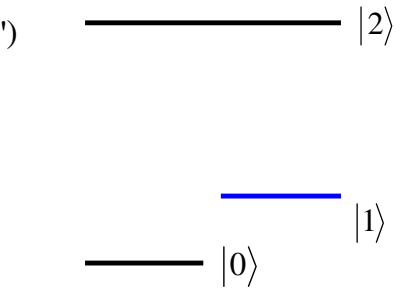

(b')

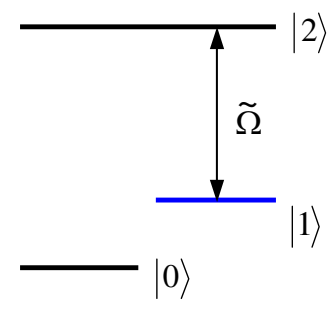

(c')

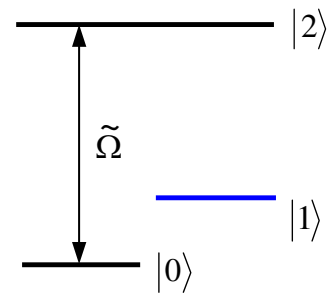

(d')

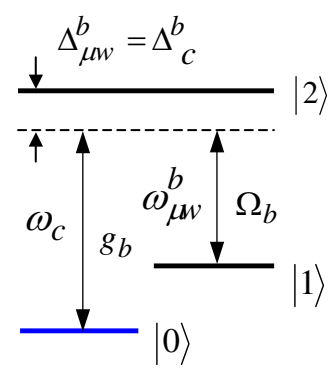

FIG. 3: (Color online) Illustration of qubits interacting with the resonator mode and/or the microwave pulses for each step of operations during the information transfer operation. Figures from top to bottom correspond to the operations of steps (i) (iv), respectively. The figures on the left side correspond to qubit $a$ while the figures on the right side correspond to qubit $b$. In addition, in each figure, the blue lines represent the level population of qubits before each step of operation. Note that the nonidentical level spacings for the two qubits are caused by the nonuniform device parameters of the two qubits.

Step (i): Apply a microwave pulse (with a frequency $\omega_{\mu w}^{a}$ ) to qubit $a$ [Fig. 3(a)]. The pulse is off-resonant with the $|1\rangle \leftrightarrow|2\rangle$ transition of qubit $a$, with a detuning $\Delta_{\mu w}^{a}=\omega_{12}^{a}-\omega_{\mu w}^{a}$. To establish the resonant Raman coupling between the two levels $|0\rangle$ and $|1\rangle$, set $\Delta_{\mu w}^{a}=\Delta_{c}^{a}$. The Rabi frequency $\Omega_{a}$ of the pulse is set by $\Omega_{a}=g_{a}$, which can be achieved by adjusting the pulse intensity. It can be seen from Eq. (3) that after a pulse duration $t_{1}=\pi \Delta_{c}^{a} /\left(2 g_{a}^{2}\right)$, the state $|1\rangle_{a}|0\rangle_{c}$ for qubit $a$ and the resonator mode is transformed to the state $|0\rangle_{a}|1\rangle_{c}$. On the other hand, the state $|0\rangle_{a}|0\rangle_{c}$ remains unchanged during the pulse.

To have a qubit coupled with the resonator mode, the qubit must be in either of the levels $|0\rangle$ or the level $|2\rangle$. Note 
that qubit $b$ was initially prepared in the state $|1\rangle$ and kept in the same state $|1\rangle$ during this step. Therefore, qubit $b$ is decoupled from the resonator mode during this step.

Step (ii): Apply a microwave pulse (with a frequency $\omega_{\mu w}^{a}=\omega_{02}^{a}$ and a phase $\phi=-\pi / 2$ ) to qubit $a$ [Fig. 3(b)] and a microwave pulse (with a frequency $\omega_{\mu w}^{b}=\omega_{12}^{b}$ and a phase $\phi=-\pi / 2$ ) to qubit $b$ [Fig. 3(b')]. The Rabi frequency for each pulse is $\widetilde{\Omega}$. Thus, it can be seen from Eq. (5) that after the pulse duration $t_{2}=\pi /(2 \widetilde{\Omega})$, the state $|0\rangle$ of qubit $a$ is transformed to the state $|2\rangle$ while the state $|1\rangle$ of qubit $b$ is transformed to the state $|2\rangle$.

Step (iii): Apply a microwave pulse (with a frequency $\omega_{\mu w}^{a}=\omega_{12}^{a}$ and a phase $\phi=\pi / 2$ ) to qubit $a$ [Fig. 3(c)] and a microwave pulse (with a frequency $\omega_{\mu w}^{b}=\omega_{02}^{b}$ and a phase $\phi=\pi / 2$ ) to qubit $b$ [Fig. 3(c')]. The Rabi frequency for each pulse is $\widetilde{\Omega}$. It can be found from Eq. (5) that after the pulse duration $t_{3}=\pi /(2 \widetilde{\Omega})$, the state $|2\rangle$ of qubit $a$ is transformed to the state $|1\rangle$ while the state $|2\rangle$ of qubit $b$ is transformed to the state $|0\rangle$.

Step (iv): Apply a microwave pulse (with a frequency $\left.\omega_{\mu w}^{b}\right)$ to qubit $b\left[\right.$ Fig. $\left.3\left(\mathrm{~d}^{\prime}\right)\right]$. The pulse is off-resonant with the $|1\rangle \leftrightarrow|2\rangle$ transition of qubit $b$, with a detuning $\Delta_{\mu w}^{b}=\omega_{12}^{b}-\omega_{\mu w}^{b}=\Delta_{c}^{b}\left[\right.$ Fig. $\left.3\left(\mathrm{~d}^{\prime}\right)\right]$. The Rabi frequency $\Omega_{b}$ of the pulse is set by $\Omega_{b}=g_{b}$. It can be seen from Eq. (3) that after a pulse duration $t_{4}=\pi \Delta_{c}^{b} /\left(2 g_{b}^{2}\right)$, the state $|0\rangle_{b}|1\rangle_{c}$ for qubit $b$ and the resonator mode is transformed to the state $|1\rangle_{b}|0\rangle_{c}$. On the other hand, the state $|0\rangle_{b}|0\rangle_{c}$ remains unchanged during the pulse.

The states of the whole system after each step of the above operations are summarized in the following table:

$$
\begin{aligned}
& |01\rangle|0\rangle_{c} \stackrel{\text { Step(i) }}{\longrightarrow}|01\rangle|0\rangle_{c} \quad \text { Step(ii) }|22\rangle|0\rangle_{c} \stackrel{\text { Step(iii) }}{\longrightarrow}|10\rangle|0\rangle_{c} \stackrel{\text { Step(iv) }}{\longrightarrow}|10\rangle|0\rangle_{c}
\end{aligned}
$$

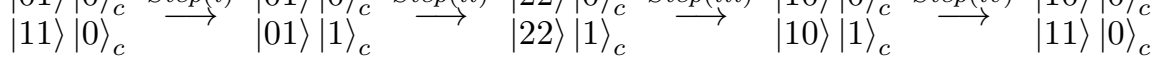

where $|i j\rangle$ is abbreviation of the state $|i\rangle_{a}|j\rangle_{b}$ of qubits $(a, b)$ with $i, j \in\{0,1,2\}$. It can be found from Eq. (8) that the transformation (7) was achieved with two qubits after the above process. Namely, the information originally carried by qubit $a$ is transferred to qubit $b$ while the resonator mode returns to its original vacuum state.

From the description above, it can be seen that:

(a) Compared with the previous proposal [8], the method presented here does not require adjustment of the level spacings of each qubit during the operation, and thus decoherence caused by the tuning of the level spacings of the qubits is avoided;

(b) Compared with the previous proposals [7,9-11], the present method does not require slow variation of the Rabi frequency, and thus the operation is speeded up;

(c) In contrast to the previous proposal [12], this method does not require a finite second-order detuning $\delta_{a}=$ $\Delta_{c}^{a}-\Delta_{\mu w}^{a}$ or $\delta_{b}=\Delta_{c}^{b}-\Delta_{\mu w}^{b}$, and thus the operation can be performed faster by one order;

(d) The level spacings for the two qubits do not need to be identical, therefore the nonuniformity in the device parameters is tolerable.

Discussion.-The occupation probability $p_{2, a}$ of the level $|2\rangle$ for qubit $a$ during step (i) and the occupation probability $p_{2, b}$ of the level $|2\rangle$ for qubit $b$ during step (iv) are given by [20]

$$
\begin{aligned}
& p_{2, a} \simeq \frac{1}{2}\left(\frac{4 \Omega_{a}^{2}}{4 \Omega_{a}^{2}+\left(\Delta_{\mu w}^{a}\right)^{2}}+\frac{4 g_{a}^{2}}{4 g_{a}^{2}+\left(\Delta_{c}^{a}\right)^{2}}\right), \\
& p_{2, b} \simeq \frac{1}{2}\left(\frac{4 \Omega_{b}^{2}}{4 \Omega_{b}^{2}+\left(\Delta_{\mu w}^{b}\right)^{2}}+\frac{4 g_{b}^{2}}{4 g_{b}^{2}+\left(\Delta_{c}^{b}\right)^{2}}\right) .
\end{aligned}
$$

The occupation probabilities $p_{2, a}$ and $p_{2, b}$ need to be negligibly small in order to reduce the operation error. For the choice of $\Delta_{\mu w}^{a}=\Delta_{c}^{a}=10 g_{a}, \Delta_{\mu w}^{b}=\Delta_{c}^{b}=10 g_{b}, \Omega_{a}=g_{a}$, and $\Omega_{b}=g_{b}$, we have $p_{2, a}, p_{2, b} \sim 0.04$, which can be further reduced by increasing the ratio of $\Delta_{c}^{a} / g_{a}, \Delta_{c}^{a} / g_{b}, \Omega_{a} / \Delta_{a}$, and $\Omega_{b} / \Delta_{b}$.

The level $|2\rangle$ of each qubit is only occupied in steps (ii) and (iii). Because resonant pulses are applied in these steps, the pulse durations $t_{2}$ for step (ii) and $t_{3}$ for step (iii) can be reduced by increasing the pulse Rabi frequencies, such that $t_{2} \ll T_{2}$ and $t_{3} \ll T_{2}$ (where $T_{2}$ is the spontaneous time of the level $|2\rangle$ of the qubits). In this way, spontaneous emission from the level $|2\rangle$ can be suppressed.

The levels $|0\rangle$ and $|2\rangle$ of qubit $a$ are populated during the pulse for step (ii) [Fig. 3(b)] and the level $|2\rangle$ of qubit $a$ is populated during the pulse for step (iii) [Fig. 3(c)]. Thus, when the resonator mode is in the single-photon state $|1\rangle_{c}$, the off-resonant interaction between the resonator mode and the $|0\rangle \leftrightarrow|2\rangle$ transition of qubit $a$ induces a phase shift $\exp \left(i t_{2} g_{a}^{2} / \Delta_{c}^{a}\right)\left[\exp \left(-i t_{2} g_{a}^{2} / \Delta_{c}^{a}\right)\right]$ to the state $|0\rangle(|2\rangle)$ of qubit $a$ for step (ii) and $\exp \left(-i t_{3} g_{a}^{2} / \Delta_{c}^{a}\right)$ to the state $|2\rangle$ of qubit $a$ for step (iii). In addition, the level $|2\rangle$ of qubit $b$ is populated during the pulse for step (ii) [Fig. 3( $\left.\left.\mathrm{b}^{\prime}\right)\right]$ and the levels $|0\rangle$ and $|2\rangle$ of qubit $b$ are populated during the pulse for step (iii) [Fig. 3( $\left.\mathrm{c}^{\prime}\right)$ ]. Hence, when the resonator mode is in the single-photon state $|1\rangle_{c}$, the off-resonant interaction between the resonator mode and the $|0\rangle \leftrightarrow|2\rangle$ 


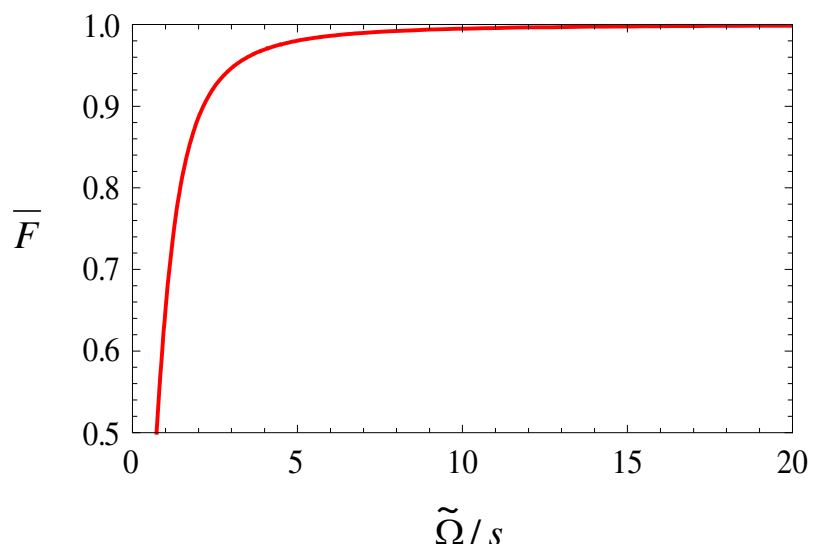

FIG. 4: Average fidelity $\bar{F}$ as a function of the Rabi frequency $\widetilde{\Omega}$ (in unit of $s$ ).

transition of qubit $b$ induces a phase shift $\exp \left(-i t_{2} g_{b}^{2} / \Delta_{c}^{b}\right)$ to the state $|2\rangle$ of qubit $b$ for step (ii) and $\exp \left(i t_{3} g_{b}^{2} / \Delta_{c}^{b}\right)$ $\left[\exp \left(-i t_{3} g_{b}^{2} / \Delta_{c}^{b}\right)\right]$ to the state $|0\rangle(|2\rangle)$ of qubit $b$ for step (iii). These phase shifts, which are not considered in Eq. (8), will affect the desired information transfer performance. However, note that $t_{2}, t_{3} \propto 1 / \widetilde{\Omega}$. Thus, these unwanted phase shifts can be made negligibly small, by increasing the pulse Rabi frequencies such that $\widetilde{\Omega} \gg g_{a}^{2} / \Delta_{c}^{a}, g_{b}^{2} / \Delta_{c}^{b}$. To see this more clearly, we will give an analysis on the effect of the unwanted qubit-resonator off-resonant interaction on the fidelity of the QIT.

In the ideal case, it can be seen from Eq. (8) that after the operations described above, the state of the two qubits and the resonator mode is $\left|\psi_{i d}(\tau)\right\rangle=|1\rangle_{a}\left(\alpha|0\rangle_{b}+\beta|1\rangle_{b}\right) \otimes|0\rangle_{c}$. Here, $\tau$ is the total operation time. On the other hand, when the off-resonant interaction between the resonator mode and the $|0\rangle \leftrightarrow|2\rangle$ transition of each qubit is included during steps (ii) and (iii), one can easily work out the expression for the final state $|\psi(\tau)\rangle$ of the whole system after performing the operations above. To simplify our presentation, we will not give a complete expression for $|\psi(\tau)\rangle$ due to its complexity. A simple calculation shows that the fidelity for the QIT is

$$
\begin{aligned}
F & =\left|\left\langle\psi_{\text {id }}(\tau) \mid \psi(\tau)\right\rangle\right|^{2} \\
& =\left(|\alpha|^{2}+p q|\beta|^{2}\right),
\end{aligned}
$$

where

$$
\begin{aligned}
& p=\frac{\widetilde{\Omega}}{\sqrt{\widetilde{\Omega}^{2}+s_{a}^{2} / 4}} \sin \left[\frac{\pi \sqrt{\widetilde{\Omega}^{2}+s_{a}^{2} / 4}}{2 \widetilde{\Omega}}\right], \\
& q=\frac{\widetilde{\Omega}}{\sqrt{\widetilde{\Omega}^{2}+s_{b}^{2} / 4}} \sin \left[\frac{\pi \sqrt{\widetilde{\Omega}^{2}+s_{b}^{2} / 4}}{2 \widetilde{\Omega}}\right],
\end{aligned}
$$

with $s_{a}=2 g_{a}^{2} / \Delta_{c}^{a}$ and $s_{b}=2 g_{b}^{2} / \Delta_{c}^{b}$.

Defining $\alpha=\cos \frac{\vartheta}{2}$ and $\beta=e^{i \varphi} \sin \frac{\vartheta}{2}$, where $\vartheta \in[0, \pi]$ and $\varphi \in[0,2 \pi]$. Thus, the average fidelity over all possible initial states of the message qubit $a$ is given by

$$
\begin{aligned}
\bar{F} & =\int_{0}^{2 \pi} d \varphi \int_{0}^{\pi} F \sin \vartheta d \vartheta / 4 \pi \\
& =\frac{1}{3}\left(1+p^{2} q^{2}+p^{4} q^{4}\right) .
\end{aligned}
$$

It can be verified that when the unwanted qubit-resonator off-resonant interaction in steps (ii) and (iii) is not considered (i.e., the case for $g_{a}^{2} / \Delta_{c}^{a}, g_{b}^{2} / \Delta_{c}^{b}=0$ or $s_{a}, s_{b}=0$ ), we have $p=q=1$, leading to $F=1$ and $\bar{F}=1$. We have plotted the average fidelity $\bar{F}$ as a function of $\widetilde{\Omega} / s$ for the choice of $s_{a}=s_{b}=s$ (Fig. 4). One can see from Fig. 4 that the average fidelity $\bar{F}$ increases as the pulse Rabi frequency $\widetilde{\Omega}$ becomes larger, and the $\bar{F}$ is $\sim 1$ when $\widetilde{\Omega}=10 \mathrm{~s}$. This result demonstrates that the effect of the qubit-resonator off-resonant interaction in steps (ii) and (iii) on the fidelity of the operation is negligible when the pulse Rabi frequencies are sufficiently large. 
Finally, let us give a rough estimate on the operation time. As shown above, the total operation time for the information transfer is

$$
\tau=\pi \Delta_{c}^{a} /\left(2 g_{a}^{2}\right)+\pi \Delta_{c}^{b} /\left(2 g_{b}^{2}\right)+\pi / \widetilde{\Omega}
$$

Without loss of generality, let us consider $g_{a} \sim g_{b} \sim 3.0 \times 10^{9} \mathrm{~s}^{-1}$, which is available at present [21]. By choosing $\Delta_{c}^{a}=10 g_{a}, \Delta_{c}^{b}=10 g_{b}$, and $\widetilde{\Omega} \sim 10 g_{a}$, we have $\tau \sim 11 \mathrm{~ns}$.

Conclusion.-We have proposed a way for realizing the quantum information transfer with superconducting flux qubits coupled to a resonator. As shown above, this proposal avoids most of the problems existing in the previous proposals. Finally, it is noted that the method presented here is quite general, which can be applied to the other physical systems such as atoms and quantum dots with the $\Lambda$-type three-level structure within cavity QED.

Acknowledgments.- This work was supported by the National Natural Science Foundation of China under Grant No. 11074062 and the starting research funds from Hangzhou Normal University.

[1] Y. Yu, S. Han, X. Chu, S. I. Chu, and Z. Wang, Science 296, 889 (2002).

[2] I. Chiorescu, P. Bertet, K. Semba, Y. Nakamura, C. J. P. M. Harmans, and J. E. Mooij, Nature (London) 431,159 (2004).

[3] J. B. Majer, F. G. Paauw, A. C. J. TerHaar, C. J. P. M. Harmans, and J. E. Mooij, Phys. Rev. Lett. 94, 090501 (2005).

[4] A. Blais, R. S. Huang, A. Wallraff, S. M. Girvin, and R. J. Schoelkopf, Phys. Rev. A 69, 062320 (2004).

[5] M. A. Sillanp, J. I. Park, and R. W. Simmonds, Nature (London) 449, 438 (2007).

[6] J. Majer, J. M. Chow, J. M. Gambetta, J. Koch, B. R. Johnson, J. A. Schreier, L. Frunzio, D. I. Schuster, A. A. Houck, and A. Wallraff et al., Nature (London) 449, 443 (2007).

[7] C. P. Yang, S. I. Chu, and S. Han, Phys. Rev. Lett. 92, 117902 (2004).

[8] C. P. Yang, S. I. Chu, and S. Han, Phys. Rev. A 67, 042311 (2003).

[9] Z. Kis and E. Paspalakis, Phys. Rev. B 69, 024510 (2004).

[10] E. Paspalakis and N. J. Kylstra, J. Mod. Opt. 51, 1679 (2004).

[11] Z. B. Feng, Z. L. Cai, C. Zhang, L. Fan, and T. Feng, Opt. Commun. 283, 1975 (2010).

[12] C. P. Yang, S. I. Chu, and S. Han, J. Phys.: Condens. Matter 16, 1907 (2004).

[13] Y. X. Liu, J. Q. You, L. F. Wei, C. P. Sun, and F. Nori, Phys. Rev. Lett. 95, 087001 (2005).

[14] S. Han, J. Lapointe, and J. E. Lukens, Single-Electron Tunneling and Mesoscopic Devices (Springer-Verlag press, Berlin Heidelberg, 1991), Vol. 31, pp. 219-222.

[15] J. Clarke and F. K. Wilhelm, Nature (London) 453, 1031 (2008).

[16] M. Neeley, M. Ansmann, R. C. Bialczak, M. Hofheinz, N. Katz1, E. Lucero, A. O'Connell, H. Wang, A. N. Cleland, and J. M. Martinis, Nature Physics 4, 523 (2008).

[17] L. Wang, R. R. Puri, and J. H. Eberly, Phys. Rev. A 46, 7192 (1992).

[18] C. P. Yang, S. I. Chu, and S. Han, Phys. Rev. A 70, 044303 (2004).

[19] J. Q. You and F. Nori, Phys. Today 58 (11), 42 (2005).

[20] C. P. Yang, Y. X. Liu, and F. Nori, Phys. Rev. A 81, 062323 (2010).

[21] C. P. Yang and S. Han, Phys. Rev. A 72, 032311 (2005). 